\title{
Evidence for Basal-Bolus Insulin Versus Slide Scale Insulin
}

\author{
Sameer Badlani • William T. Ford Jr. • \\ David J. Yu • Gerard X. Brogan Jr. • \\ Charles V. Pollack Jr. • Gregory A. Volturo
}

Published online: 7 March 2014

(c) Springer Science+Business Media New York 2014

\begin{abstract}
Greater understanding of hyperglycemia and its control in non-ICU patients has become ever more urgent given the high and increasing prevalence of diabetes in the general population and, hence, in hospitalized patients. It is well accepted that hyperglycemia in hospitalized patients is common and associated with profound medical consequences, longer lengths of stay, high healthcare costs, and adverse outcomes. It is a marker for poor clinical outcome and mortality. Although evidence that supports intensive glycemic control in critically ill patients is strong, glycemic control is often overlooked or insufficient in patients on general medicine and surgery services. In the face of strong
\end{abstract}

S. Badlani $(\square)$

Section of Hospital Medicine, University of Chicago Medicine,

Chicago, IL, USA

e-mail: sbadlani@medicine.bsd.uchicago.edu

W. T. Ford Jr.

Temple University School of Medicine, Philadelphia, PA, USA

D. J. Yu

Adult Inpatient Medicine Service, Presbyterian Medical Group, Albuquerque, NM, USA

G. X. Brogan Jr.

Department of Emergency Medicine, Hofstra North Shore LIJ

School of Medicine, Hempstead, NY, USA

C. V. Pollack Jr.

Department of Emergency Medicine, Pennsylvania Hospital,

University of Pennsylvania, Philadelphia, PA, USA

G. A. Volturo

Emergency Medicine and Medicine, University of Massachusetts

Medical School, Worcester, MA, USA evidence that glycemic control helps to improve outcomes in non-ICU patients, it is critical to consider how best to manage hyperglycemia in medical and surgical patients to develop optimum strategies for maintaining glycemic control. Currently available strategies for glycemic control include sliding-scale insulin and basal-bolus regimens. The principal difference between the two strategies is that sliding-scale insulin does not deliver adequate glycemic control to patients and addresses hyperglycemia after it has occurred, whereas a basal-bolus regimen is directed at preventing hyperglycemia. This paper explores the rationale for and implementation of a basal-bolus insulin regimen in non-critically ill hospitalized patients and in addition reviews best practices for transitions of care and discharge planning.

Keywords Diabetes - Slide scale insulin - Basal-bolus insulin · Diabetes and insulin - Hospitalization for diabetes $\cdot$ Emergency and hospital medicine

\section{Introduction}

Hyperglycemia in hospitalized patients is a common occurrence with potentially serious and profound medical consequences, longer lengths of stay and high associated healthcare costs [1•]. In fact, hyperglycemia is correlated with adverse outcomes and can occur in hospitalized patients with known or undiagnosed diabetes [ $1 \bullet]$.

Evidence that the onset of hyperglycemia during acute medical or surgical illness is not a physiologic or benign condition is increasing. Rather, the development of hyperglycemia is a marker of poor clinical outcome and mortality $[1 \bullet$. 


\section{Diabetes and the Hospital Experience}

Much of what is known about hyperglycemia in hospitalized patients has come from studies of patients in the ICU; information about optimal management of hyperglycemia in the non-ICU setting is limited. Greater understanding of hyperglycemia and its control in non-ICU patients is urgent as the prevalence diabetes is reaching epidemic proportions.

As of 2007, approximately 23 million individuals in the United States had diabetes, accounting for $22 \%$ of all hospital inpatient days. A total economic burden of $\$ 174$ billion was associated with their care and almost half of which was spent in an inpatient setting [3].

\section{Rationale for Improved Glycemic Control}

The rationale for improved glycemic control in medical and surgical non-ICU patients is strong. Data from observational studies suggest that in surgical patients, with and without diabetes, an improvement in glycemic control exerts positive effects on morbidity and mortality. Evidence from the cardiac surgery and surgical intensive care settings demonstrates perioperative hyperglycemia is associated with an increased rate of deep sternal wound infections, hospital complications and the improvement in glycemic control is associated with reduced rate of postoperative complications, length of hospital stay, and mortality $[1 \cdot]$.

In addition, the development of perioperative hyperglycemia in general surgery patients has been seen as a sensitive predictor of nosocomial infection in small observational studies. To date, few studies have addressed the association between glucose levels and hospital mortality in general surgery patients; hence, there is no information whether the severity of hyperglycemia and the timing of hyperglycemia perioperative period are factors that contribute to the increased mortality and hospital complications that have been observed in cardiac surgery and surgical ICU patients [4].

\section{ADA Recommendations for Treating Hyperglycemia}

According to the American Diabetes Association (ADA), substantial observational evidence links hyperglycemia to poor outcomes in hospitalized patients. In 2013, the ADA added recommendations for treatment of hyperglycemia in hospitalized patients to its annual Standards of Medical Care [1•]. According to the ADA guidelines, insulin therapy is the preferred method of glycemic control for the majority of in-hospital clinical situations. Intravenous infusion is the preferred route of insulin administration in the intensive care unit (ICU). In other hospital settings (including the ED), the standards recommend scheduled subcutaneous insulin that delivers basal, nutritional, and correction (supplemental) components. Typical dosing schemes are based on body weight, with some evidence that patients with renal insufficiency should be treated with lower doses [5]. The remainder of this paper will discuss basal insulin (basal-bolus regimen) versus sliding-scale insulin (SSI) in non-ICU hospitalized patients.

\section{Sliding Scale Insulin (SSI) Regimens}

SSI was the standard or care for hyperglycemic hospitalized patients until the 1970s, when dosage adjustment could be made on the basis of blood glucose measurements. Today, SSI remains one of the most common strategies for managing hyperglycemia in hospitalized patients $[1 \bullet]$. Although evidence that supports intensive glycemic control in critically ill patients is strong and increasing, glucose control is often overlooked or insufficient in patients on general medicine and surgery services. View Table 1 for an example of factors that may account for the lack of glycemic control in hospitalized patients.

\section{Disadvantages and Limitations of SSI Regimens}

Although the potential advantages of SSI include convenience, simplicity, and prompt treatment, there is a paucity of evidence that it is associated with improved clinical outcomes when used as a sole source of insulin in hospitalized patients.

Prolonged therapy with SSI as the sole regimen is ineffective in the majority of patients, increases risk of both hypoglycemia and hyperglycemia, and has recently been shown in a randomized trial to be associated with adverse outcomes in general surgery patients with type 2 diabetes [1•] Note that SSI is potentially dangerous in type 1 diabetes [2]. See Table 2 for examples of limitations of SSI.

Table 1 Factors that may account for lack of glycemic control in hospitalized patients $\left[1^{\bullet}\right]$

Physicians may perceive hyperglycemia as a consequence of stress and acute illness and may defer addressing it until blood glucose levels exceed $200 \mathrm{mg} / \mathrm{dl}$.

Fear of hypoglycemia is one of the principal barriers to initiating measures to improve glycemic control, particularly in patients with very low caloric intake.

Inpatient physicians often do not order the patients' outpatient regimens and initiate sliding-scale coverage with regular insulin instead. 
Table 2 Limitations of Sliding Scale Insulin

Treats hyperglycemia after it has occurred rather than preventing its development

Associated with adverse outcomes in general surgery patients with type 2 diabetes

Potentially dangerous in patients with type 1 diabetes

SSI use is associated with limited therapeutic success and suboptimal glycemic control

Use of SSI does not deliver physiologic insulin levels and is associated with wide swings in blood glucose levels that may be more detrimental to patients than elevated BG

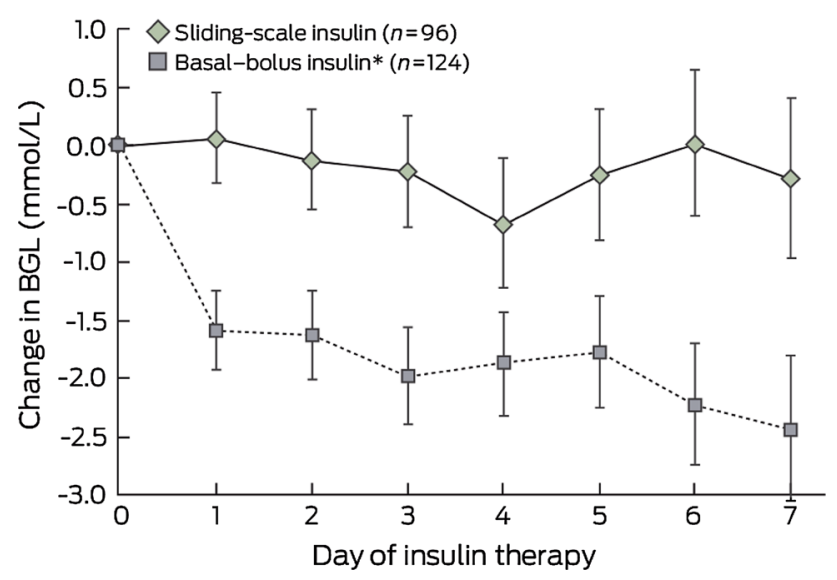

Fig. 1 Median change in blood glucose level from baseline in the two insulin therapy groups. ${ }^{*} P<0.001$ versus baseline blood glucose for all days of basal-bolus insulin therapy [7•]

\section{Basal-Bolus Insulin (BBI) Regimens}

Basal insulin differs markedly from traditional SSI in both its administration and effects on blood glucose. Figure 1 shows median change in blood glucose level from baseline blood glucose level in a group that received SSI compared with a group that received a BBI regimen [1•]. Table 3 summarizes various insulin types with their brand names, duration of action and peak of onset of effect. See Fig. 2 for physiological principles for BBI and Table 5 for components of BBI.

\section{The Case for a BBI Regimen}

The rationale for use of a BBI regimen is supported by results from several clinical studies. The RABBIT 2 study, the first prospective randomized clinical trial that compared the efficacy and safety of a basal-bolus regimen with that of SSI in non-critically ill patients with type 2 diabetes, was published in 2007. It enrolled 130 insulin-naïve patients, admitted to general medicine services, who were randomized to two closely-matched groups with respect to age, race, BMI, BG on admission, and hemoglobin A1c. Sixty-five patients received insulin glargine (basal insulin) and glulisine (bolus insulin) and sixty-five were randomized to SSI as the sole treatment. The treatment regimen is shown in Table $4\left[1^{\bullet}\right]$.

The most common admitting illnesses in RABBIT 2 included cardiovascular conditions $(40 \%)$, infectious conditions $(20 \%)$, pulmonary conditions $(18 \%)$, renal conditions $(4 \%)$ and gastrointestinal disorders (12\%). Although mean durations of hospital stay were similar, patients who received insulin glargine and glulisine exhibited greater improvement in glycemic control compared with those who received SSI alone $(P<0.01)$, as shown in Fig. 3. Mean glucose concentration was significantly higher among patients treated with SSI compared with patients who received the basal-bolus regimen (187 vs $140 \mathrm{mg} / \mathrm{dl}, P<0.001)$. See Fig. 3 for changes in bood glucose concentrations in patients treated with glargine plus glulisine and with SSI.

The overall difference between treatment groups was $27 \mathrm{mg} / \mathrm{dl}$ and the mean difference in daily blood glucose ranged from 23 to $58 \mathrm{mg} / \mathrm{dl} .66 \%$ of patients who received glargine and glulisine achieved a mean glucose target of $<140 \mathrm{mg} / \mathrm{dl}$ compared with only $38 \%$ who received SSI $[1 \bullet]$.

The investigators also established that mean blood glucose concentrations in subjects with persistent hyperglycemia despite increasing doses of SSI improved after initiation of the basal-bolus regimen, as shown in Fig. 4 $[1 \bullet]$.

Hypoglycemia, which was defined in this study as a blood glucose $<60 \mathrm{mg} / \mathrm{dl}$, occurred in two patients in each group. Of the 1,005 glucose readings in the insulin glargine and glulisine treatment group, four $(0.4 \%)$ glucose values $<60 \mathrm{mg} / \mathrm{dl}$ were noted, and none of $<40 \mathrm{mg} / \mathrm{dl}$ were seen. Of the 1,021 glucose readings in the SSI group, there were two $(0.2 \%)$ glucose values $<60 \mathrm{mg} / \mathrm{dl}$ and no glucose values $<40 \mathrm{mg} / \mathrm{dl}$. Oral glucose was given to address hypoglycemia, and no episode of hypoglycemia were associated with adverse outcomes [1•].

The investigators concluded that the BBI algorithm using insulin glargine once daily and insulin glulisine before meals represents a simple and more effective regimen than SSI for glucose control in non-critically ill patients with type 2 diabetes.

Results of later studies support the conclusion that a basal-bolus regimen delivers glycemic control compared with SSI in non-ICU hospitalized patients.

The RABBIT 2 Surgery study, a prospective randomized trial published in 2011 analyzed results from 211 adult patients who were admitted to undergo general elective or emergency surgery and were not expected to require 
Table 3 Insulin types, brand names, onset, peak, and duration of action

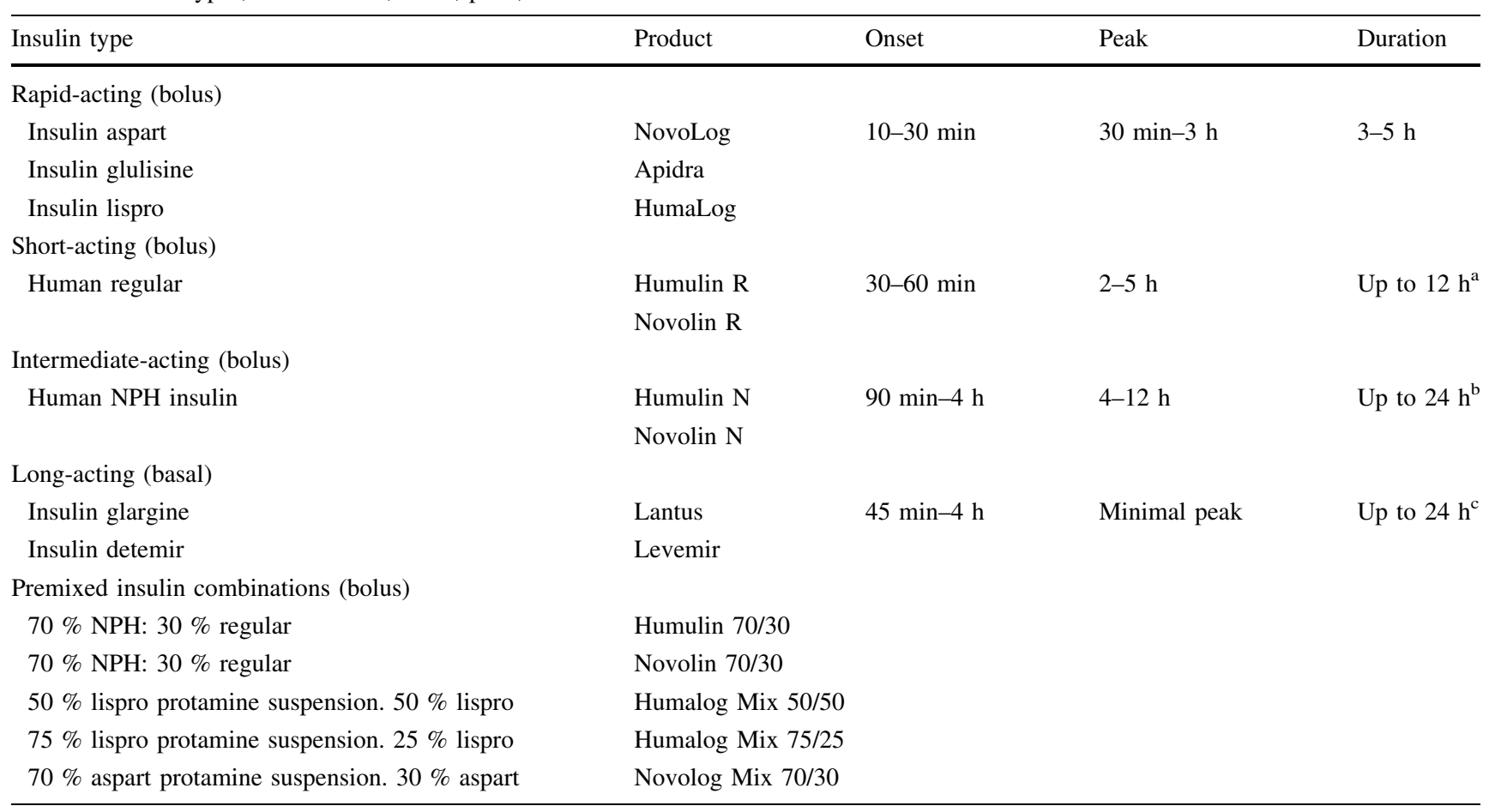

${ }^{a}$ Usual clinical relevance can be less than $12 \mathrm{~h}$

b Usual clinical relevance can be less than $24 \mathrm{~h}$. Often requires twice daily dosing

c Individual response may require twice daily dosing

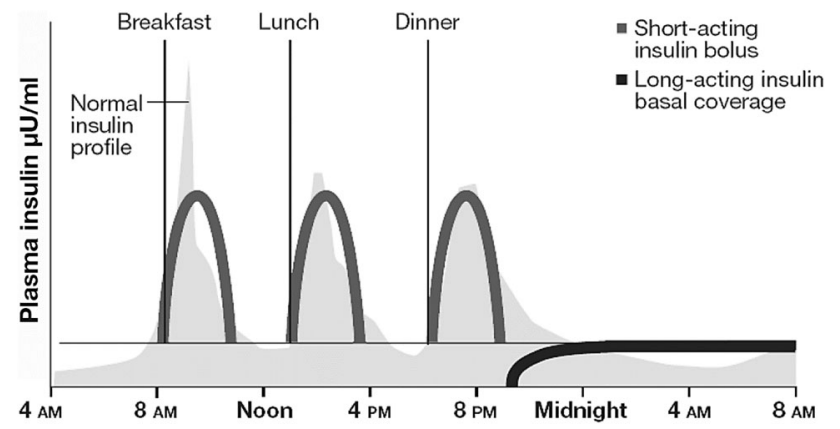

Fig. 2 Physiologic principles of the BBI [8]

admission to the ICU. Eligibility criteria included blood glucose (BG) level between 140 and $400 \mathrm{mg} / \mathrm{dl}$ and a history of diabetes for $>3$ months, age 18-80, treated with diet alone, any combination of oral antidiabetic agents, or low-dose insulin therapy at a daily dose $\leq 0.4$ units $/ \mathrm{kg}$ before admission [6].

Exclusion criteria included hyperglycemia without a known history of diabetes, cardiac surgery, clinically relevant hepatic disease or impaired renal function (serum creatinine $\geq 3.0 \mathrm{mg} / \mathrm{dl}$ ), history of diabetic ketoacidosis, pregnancy, and any mental condition that precluded giving informed consent. A total of 104 patients received a basal-
Table 4 Correction/ Supplemental Insulin Scales [1•]

\begin{tabular}{llll}
\hline & Insulin sensitive & Usual & Insulin resistant \\
\hline $\begin{array}{llll}\text { Blood glucose (mg/dl) } \\
>141-180\end{array}$ & 2 & 4 & 6 \\
$1-220$ & 4 & 6 & 8 \\
$221-260$ & 6 & 8 & 10 \\
$261-300$ & 8 & 10 & 12 \\
$301-350$ & 10 & 12 & 14 \\
$351-400$ & 12 & 14 & 16 \\
$>400$ & 14 & 16 & 18
\end{tabular}

The numbers in each column indicate the number of units of glulisine or regular insulin per dose. Supplemental dose is to be added to the scheduled dose of glulisine or regular insulin

bolus regimen, and 107 received SSI only, with a goal of maintaining fasting and premeal glucose concentration between 100 and $140 \mathrm{mg} / \mathrm{dl}$. Oral antidiabetic drugs were discontinued on admission [6].

Patients treated with basal-bolus therapy were started at a total daily dose (TDD) of 0.5 units/ $\mathrm{kg}$ divided into half as insulin glargine once daily and the other half as insulin glulisine given before meals. Patients who were unable to eat received insulin glargine, but insulin glulisine was withheld until meals were resumed. In patients $\geq 70$ years 
Table 5 Components of a basal/bolus regimen

The three components of a basal/bolus regimen are basal insulin, meal or nutritional bolus insulin, and correction insulin

The ideal basal insulin provides a constant 24-h "peakless," or tonic insulin level to suppress hepatic glucose release during the fasting state and between meals. Glargine and detemir deliver relatively peakless basal insulin. Glargine is preferred because it has a longer duration of action and permits true once-daily administration. When used appropriately, basal insulin should not cause hypoglycemia in patients who are restricted from oral nutritional intake (NPO)

The purpose of mealtime bolus insulin is to prevent predictable postprandial rise in glucose. Thus, bolus insulin can be given at each meal with one of the rapid-acting analogs (lispro, aspart, or glulisine). These insulin analogs offer rapid onset of action and typically reach peak levels within $60 \mathrm{~min}$. Rapid-acting insulin analogs should be administered within 15 min before a meal, but regular insulin, due to its slower onset of action, must be given at least $30 \mathrm{~min}$ before a meal

The purpose of correction insulin is to lower hyperglycemic glucose levels rather than cover nutritional hyperglycemia. However, in common with mealtime bolus insulin, rapid-acting analog formulations are a good choice for correctional insulin for patients who can eat [8]

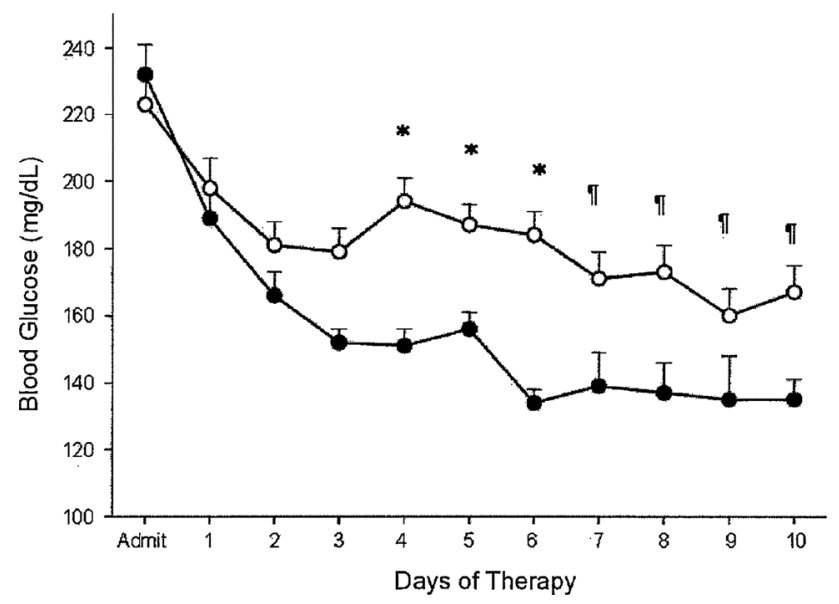

Fig. 3 Changes in blood glucose concentrations in patients treated with glargine plus glulisine and with SSI [2]. filled circle glargine plus glulisine, circle SSI

of age and or with serum creatinine $\geq 2 \mathrm{mg} / \mathrm{dl}$, insulin TDD was reduced to 0.3 units $/ \mathrm{kg}$. Patients randomized to SSI received regular insulin four times daily for $B G>140 \mathrm{mg} /$ dl. Insulin doses were adjusted according to a prespecified protocol. Patients in the SSI group were switched to the basal-bolus regimen starting at a TDD of 0.5 units $/ \mathrm{kg}$ if their mean daily BG level was $>240 \mathrm{mg} / \mathrm{dl}$ or if three consecutive values $>240 \mathrm{mg} / \mathrm{dl}$ on the maximal sliding scale dose were noted [6].

Results were similar to those in the 2007 RABBIT 2 study, as shown in Fig. 5.

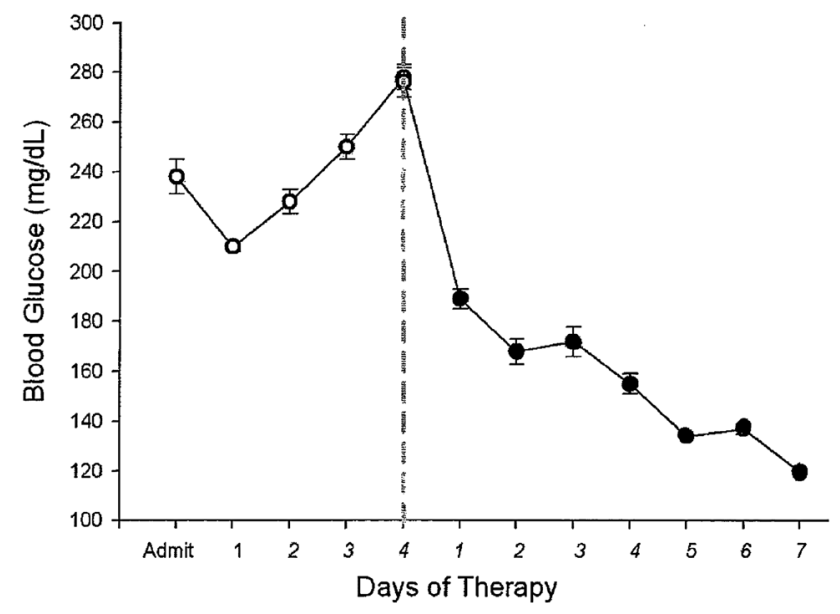

Fig. 4 Mean blood glucose concentration in subjects who remained with severe hyperglycemia despite increasing doses of regular insulin per the sliding-scale protocol (circle). Glycemic control rapidly improved after switching to the BBI regimen (filled circle) [1•]

Hypoglycemia $(<70 \mathrm{mg} / \mathrm{dl})$ occurred in $23.1 \%$ of patients in the basal-bolus and $4.7 \%$ of patients in the SSI treated group $(P<0.001)$. Severe hypoglycemia $(<40 \mathrm{mg} /$ dl) was reported in $3.8 \%$ of patients in the basal-bolus and none in the SSI group $(P=0.057)$. There were no differences in the frequency of hypoglycemia between patients treated with insulin before admission compared with insulin-naïve patients. A total of 15 patients $\geq 70$ years of age or with a serum creatinine $\geq 2 \mathrm{mg} / \mathrm{dl}$ received an initial TDD of $0.3 \mathrm{unit} / \mathrm{kg}$. There were no differences in mean daily BG or the frequency of hypoglycemic events between the 0.3 units $/ \mathrm{kg}$ and the 0.5 units $/ \mathrm{kg}$ groups [6].

In comparing the results of the RABBIT 2 medical and surgical trials, the investigators noted that, "The basalbolus regimen with glargine once daily and glulisine before meals at a starting dose of $0.3-0.5$ unit $/ \mathrm{kg} / \mathrm{day}$ is well tolerated with an acceptable rate of hypoglycemia. In the RABBIT medicine trial, only two patients $(3 \%)$ in the glargine and glulisine group experienced a BG $<60 \mathrm{mg} / \mathrm{dl}$ and no patients had a value $<40 \mathrm{mg} / \mathrm{dl}$. In this RABBIT surgery trial, a glucose $<70 \mathrm{mg} / \mathrm{dl}$ was reported in $23.1 \%$ of patients (1.9\% of glucose readings) in the basal-bolus and in $4.7 \%(0.3 \%$ of readings $)$ in the SSI group $(P=0.001)$, but there were no significant differences in the frequency of severe hypoglycemia" [6].

The investigators observe that differences in hypoglycemic events between the two trials could be the result of reduced nutritional intake in surgical patients and the fact that in the previous trial patients received the TDD of insulin as 0.4 units $/ \mathrm{kg}$ for BG between 140 and $200 \mathrm{mg} / \mathrm{dl}$ and 0.5 unit $/ \mathrm{kg}$ for BG between 200 and $400 \mathrm{mg} / \mathrm{dl}$. In contrast, most patients in the RABBIT surgery trial were given a single daily dose of 0.5 units $/ \mathrm{kg}$ [6]. 


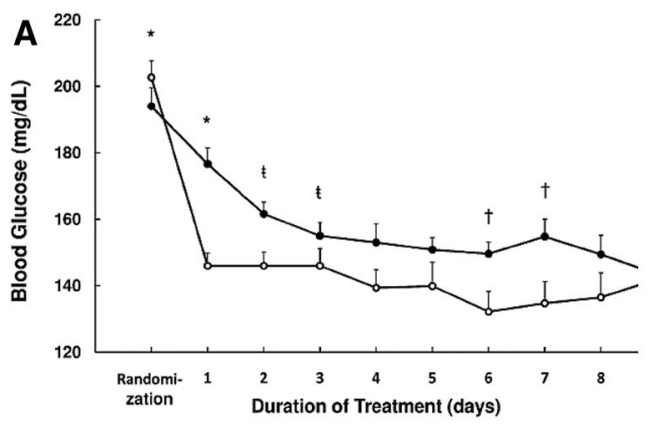

Fig. 5 a Glucose levels during basal-bolus and SSI treatment. Changes in blood glucose concentration after the first day of treatment with basal-bolus with glargine once daily plus glulisine before meals (circle) and with SSI 4-times daily (filled circle). $* P<0.001$,

Other studies supporting the findings of the RABBIT 2 medical and surgical trials include the Basal Plus Trial, BBI versus SSI for inpatient glycemic control, a clinical practice comparison by Roberts et al. [7•] and a study conducted by Gosmanov, who looked at hyperglycemic diabetic patients with malignancy on dexamethasone therapy [5].

The Basal Plus trial was a multicenter randomized trial enrolling 375 patients with type 2 diabetes who were being treated with diet, oral antidiabetic agents, or low-dose insulin (\#0.4 units/kg/day). Patients were randomized to receive a basal-bolus regimen with glargine once daily and glulisine before meals, a basal-plus regimen with glargine once daily and supplemental doses of glulisine, and a sliding scale regular insulin arm. Improvement in mean daily blood glucose (BG) after the first day of therapy was similar between basal bolus and basal plus groups $(P=0.16)$. Both regimens resulted in a lower mean daily serum glucose than did SSI $(P=0.04)$. Additionally, treatment with the basal bolus and basal plus regimens resulted in less treatment failure (defined as two consecutive glucose readings above $240 \mathrm{mg} / \mathrm{dl}$ or a mean daily glucose greater than $240 \mathrm{mg} / \mathrm{dl}$ ) than did treatment with SSI ( 0 vs. 2 vs. $19 \%$, respectively; $P=0.001$ ).

A glucose of less than $70 \mathrm{mg} / \mathrm{dl}$ occurred in $16 \%$ of patients in the basal bolus group, $13 \%$ in the basal plus group, and $3 \%$ in the SSI group $(P=0.02)$. There was no difference among the groups in the frequency of severe hypoglycemia $(<40 \mathrm{mg} / \mathrm{dl} ; P=0.76)$. Thus the authors concluded that the use of a basal plus regimen with glargine once daily plus corrective doses with glulisine insulin before meals resulted in glycemic control similar to a standard basal bolus regimen and that the basal plus approach is an effective alternative to the use of a basal bolus regimen in patients with type 2 diabetes with general medical and surgical conditions.

Roberts and colleagues performed a cross-sectional study using capillary blood glucose measurements to

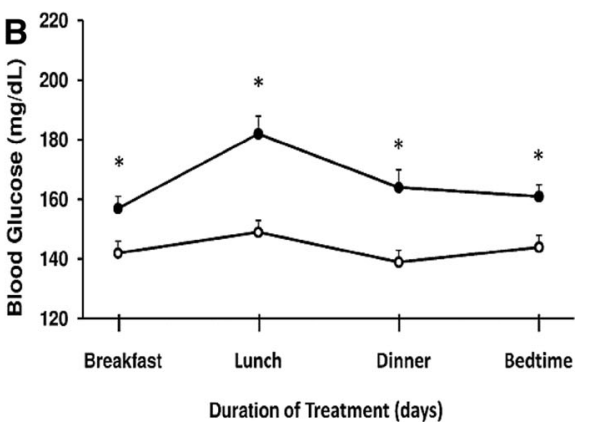

${ }^{\mathrm{t}} P=0.02,{ }^{\dagger} P=0.01$. b Glucose levels before meals and bedtime. Premeal and bedtime glucose levels were higher throughout the day in the SSI group (filled circle) compared with basal-bolus regimen (circle) [6]

determine if the improvement in inpatient glycemic control was achieved with BBI versus SSI [7•]. Capillary blood glucose levels were prospectively measured four times daily for up to eight days in 124 patients with type 2 diabetes admitted to a tertiary teaching hospital and treated with BBI.

The BBI treatment group was retrospectively compared with data from 96 patients previously treated with SSI, with the main outcome measures being mean daily blood glucose and the independent effect of the insulin regimen on mean daily blood glucose. The investigators found that there was not a significant difference in mean baseline blood glucose levels in patients receiving $\mathrm{BBI}$ and SSI (mean $\pm \mathrm{SD}$, $11.3 \pm 4.1$ vs. $10.6 \pm 4.3 \mathrm{mmol} / \mathrm{l} ; P=0.23)$. After the first full day of therapy, the mean daily blood glucose for patients receiving BBI was $1.6 \pm 3.7 \mathrm{mmol} / \mathrm{l}$ lower than baseline; and it remained 1.6-2.4 $\mathrm{mmol} / \mathrm{l}$ lower than baseline throughout the study period $(P<0.001)$. There was no significant change in blood glucose for patients receiving SSI.

A random effects regression analysis indicated that BBI was associated with significantly lower mean daily blood glucose than SSI, independent of other variables $(P<0.001)$. The incidence of hypoglycemia (glucose $<4 \mathrm{mmol} / \mathrm{l}) \quad\{72 \mathrm{mg} / \mathrm{dl}\}$ was significantly greater in patients receiving BBI than SSI $(3.3 \times 1.4 \% ; P<0.001)$, but there was no significant difference for severe hypoglycemia (glucose $<2.8 \mathrm{mmol} / \mathrm{l})\{50 \mathrm{mg} / \mathrm{dl}\}$ (0.3 vs. $0.5 \%$; $P=0.3$ ). The authors concluded that under routine clinical conditions, BBI is effective and safe across a wide range of patients and appears to be superior to SSI.

Finally, in a retrospective study to determine whether the response to a BBI regimen with detemir and aspart is superior to a SSI regimen for management of hyperglycemia in hospitalized diabetic patients receiving dexamethasone, investigators reported the results of forty patients with hematologic malignancies who were treated with intravenous ( 8 to $12 \mathrm{mg} /$ day $)$ or oral $(40 \mathrm{mg} /$ day) 
dexamethasone for three days. The average blood glucose level was $301 \pm 57 \mathrm{mg} / \mathrm{dl}$ in the SSI group $(n=28)$ and $219 \pm 51 \mathrm{mg} / \mathrm{dl}$ in the BBI group $(n=12)(P<0.001)$. The BBI regimen resulted in an average blood glucose reduction of $52 \pm 82 \mathrm{mg} / \mathrm{dl}$ throughout the course of dexamethasone therapy, while the SSI regimen produced an increase in the mean daily blood glucose level of $128 \pm 77 \mathrm{mg} / \mathrm{dl}(P<0.001)[11]$.

On the last day of dexamethasone administration, the insulin requirement was $49 \pm 29$ units/day in the SSI group and $122 \pm 39$ units/day in the BBI group $(P<0.001)$. Complications were more prevalent in the SSI group, with three patients developing diabetic ketoacidosis or hyperosmolar hyperglycemia during steroid therapy. No hypoglycemia was observed in either group. The lengths of stay and infection rates were similar between groups. Authors concluded that a basal and bolus insulin regimen is an effective and safe approach for managing dexamethasone-induced hyperglycemia in hospitalized patients with hematologic malignancies [1•].

Other trials have evaluated the use of BBI in the management of hyperglycemia in hospitalized patients who receive enteral and parenteral nutrition. Although there are no specific guidelines for glycemic targets and effective strategies for the management of hyperglycemia

Table 6 Prevention and management of hypoglycemia in patients receiving enteral and parenteral nutrition [10]

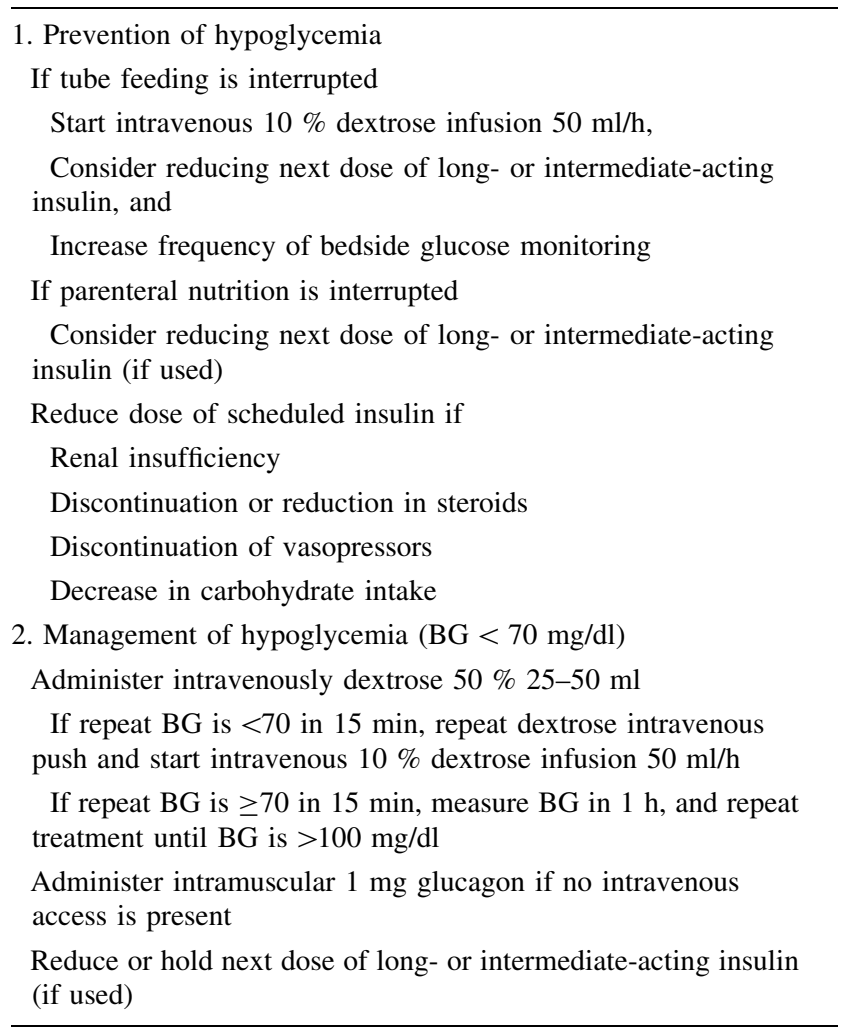

Table 7 Risk factors for hypoglycemia

Excessive dose of insulin

Discontinuation of vasopressors

Decrease in carbohydrate intake

Interruption of enteral or parenteral nutrition

during specialized nutritional support, such management is of critical concern because hyperglycemia in this group of patients is associated with an increased risk of death and infectious complications. Thus, prevention and correction of hyperglycemia should be undertaken through modification of nutrient composition or by insulin infusion. Concern about hypoglycemia may present barriers to the initiation of insulin therapy in these patients; it can occur as a result of excess insulin dose, abrupt discontinuation of nutritional support, recovery from acute illness, decreases in dose of steroids or vasopressors, and progressive organ damage. Strategies for the prevention and management of hypoglycemia in patients who receive enteral and parenteral nutrition are shown in Table 6 [10]. Also, see Table 7 for risk factors for hypoglycemia.

\section{Implementing a Basal/Bolus Insulin regimen}

Basal-Bolus Regimen with Insulin Glargine and Glulisine

1. Discontinue oral antidiabetic drugs on admission.

2. Calculate total daily insulin dose:

0.4 units/kg body wt/day when the admission blood glucose concentration is $140-200 \mathrm{mg} / \mathrm{dl}$

0.5 units/kg body wt/day when the admission blood glucose concentration is between 201 and $400 \mathrm{mg} / \mathrm{dl}$

3. Give one-half of total daily dose as insulin glargine and the other half as insulin glulisine.

4. Give insulin glargine at the same time of the day.

5. Give insulin glulisine in three equally divided doses before each meal. Hold insulin glulisine if patient is not able to eat. Give half if patient only finishes half of the meal.

6. Give correction/supplemental insulin glulisine in addition for blood glucose $>140 \mathrm{mg} / \mathrm{dl}$.

7. If a patient is able and expected to eat all or most of his/her meals, give supplemental glulisine insulin before each meal and at bedtime following the "usual" column.

8. If a patient is not able to eat, give supplemental glulisine insulin every $6 \mathrm{~h}(6-12-6-12)$, following the "insulin-sensitive" column. 
Insulin Adjustment

9. If the fasting or mean blood glucose during the day is $140 \mathrm{mg} / \mathrm{dl}$ in the absence of hypoglycemia, increase insulin glargine dose by $20 \%$ every day.

10. If patient develops hypoglycemia $(<70 \mathrm{mg} / \mathrm{dl})$, decrease glargine daily dose by $20 \%$.

Blood glucose monitoring

11. Measure blood glucose before each meal and at bedtime (or every $6 \mathrm{~h}$ if npo)

\section{Transitions of Care}

Patients transitioning from hospital to community require appropriate education and follow-up to facilitate maintenance of glycemic control and achieve glycemic goals. An organized multidisciplinary team may be best qualified to deliver transitional and ongoing care for patients with DM. Members of such a team can include a primary care physician, endocrinologist, physician assistant, nurse practitioner, registered nurse, certified diabetes educator (CDE)/ nurse educator, dietitian, exercise specialist, and a mental health care professional $[1 \bullet]$.

Patient education should be comprehensive, particularly for newly diagnosed patients, and should stress selfmanagement strategies. At diagnosis and throughout their lives, and particularly during health crises, patients should receive information and counseling about therapeutic lifestyle management that includes medical nutrition therapy (with reduction and modification of caloric and fat intake to achieve weight loss in those who are overweight or obese), appropriate physical activity, avoidance of tobacco products, and adequate quantity and quality of sleep [11].

Optimal post-discharge follow-up should occur over the long term with a primary care physician, an endocrinologist, a diabetes educator, and other health care professionals such as an ophthalmologist, a vascular specialist, a cardiologist, and a nephrologist as appropriate.

The most critical time in all of in-patient management is the transition from one medical setting to the next. The opportunity for medical errors and miscommunication increases during these transition points. Providers of hospital care should be very cautious in their approach with patients on insulin or other diabetic agents. Often times, patients are unable to completely comprehend their treatment or follow up plans post discharge. It is imperative that we communicate all information to the next care provider scheduled to care for the patient, i.e., primary care physician or endocrinologist. Communication with a patient's family or caregiver is also extremely useful in situations that involve insulin therapy. Finally, follow up education and re-education will help the patient understand their disease process and transition back to a healthy lifestyle.

\section{Summary}

Given the high and increasing prevalence of patients with diabetes, it is increasingly urgent to establish strategies and protocols for glycemic control in non-critically ill patients on medical and surgical services.

Although the potential advantages of SSI include convenience, simplicity, and prompt reduction of blood glucose levels, there is little evidence that sole use of this regimen is associated with improved clinical outcomes. In fact, use of SSI is associated with limited therapeutic success and suboptimal glycemic control due to wide springs in blood glucose levels that may be more detrimental to patients than elevated blood glucose. It is also associated with adverse outcomes in general surgery patients with type 2 diabetes and is potentially dangerous in patients with type 1 diabetes.

Despite it's simplicity, the SSI regimen fails to provide adequate glycemic control and should not be used in the management of hospitalized subjects with diabetes. Implementing standardized subcutaneous insulin order sets promoting the use of scheduled insulin therapy and discouraging the sole use of SSI are key interventions that might reduce complications associated with severe hyperglycemia and hypoglycemia in hospitalized patients.

In contrast, a basal-bolus regimen helps to prevent hyperglycemia and delivers more physiologic insulin levels throughout the day and night. Thus, the purpose of basal insulin is to provide a "base" on which to add bolus insulin at mealtime to avoid postprandial spikes in blood glucose. Meticulous transition-of-care and discharge planning should accompany hospital discharge in general and, in particular, for patients who have had elevated blood glucose while hospitalized.

\section{Compliance with Ethics Guidelines}

Conflict of Interest This paper was underwritten in part by a grant from Sanofi to the Hospital Quality Foundation to support the writing group's efforts, including travel. All authors report receiving consulting fees from Sanofi.

Human and Animal Rights and Informed Consent This article does not contain any studies with human or animal subjects performed by any of the authors. 


\section{References}

Papers of particular interest, published recently, have been highlighted as:

- Of importance

1. - Umpierrez GE, Smiley DS, Zisman A, et al. Randomized study of basal-bolus insulin therapy in the inpatient management of patients with type 2 diabetes (RABBIT 2 Trial). Diabetes Care. 2007;30:2181-6. This randomized trial showed clearly that a basal-bolus insulin regimen is preferred over SSI in the management of non-critically ill, hospitalized patients with type 2 diabetes.

2. Moghissi ES, Korytkowski MT, DiNardo M, et al. American Association of Clinical Endocrinologists and American Diabetes Association consensus statement on inpatient glycemic control. Endocr Pract. 2009;15:353-69.

3. Pichardo-Lowden AR, Fan CY, Gabbay RA. Management of hyperglycemia in the non-intensive care patient: Featuring subcutaneous insulin protocols. Endocr Pract. 2011;17:249-60.

4. Frisch A, Chandra P, Smiley D, et al. Prevalence and clinical outcome of hyperglycemia in the perioperative period in noncardiac surgery. Diabetes Care. 2010;33:1783-8.
5. American Diabetes Association. Standards of Medical Care in Diabetes-2013. Diabetes Care. 2013;36(Suppl 1):S11-66.

6. Umpierrez GE, Smiley D, Jacobs S, et al. Randomized study of basal-bolus insulin therapy in the inpatient management of patients with type 2 diabetes undergoing general surgery (RABBIT 2 surgery). Diabetes Care. 2011;34:256-61.

7. - Roberts GW, Aguilar-Loza N, Esterman A, et al. Basal-bolus insulin versus sliding-scale insulin for inpatient glycaemic control: a clinical practice comparison. MJA. 2012;196:266-9. In a similar population to RABBIT-2, this study confirmed that under routine clinical conditions, basal-bolus insulin is effective and safe across a range of patients and appears to be superior to SSI.

8. Magaji V, Johnston JM. Inpatient management of hyperglycemia and diabetes. Clin Diabetes. 2011;29:3-9.

9. Gosmanov AR, Goorha S, Stelts S, et al. Management of hyperglycemia in diabetic patients with hematologic malignancies during dexamethasone therapy. Endocr Pract. 2013;19:231-5.

10. Gosmanov AR, Umpierrez GE. Management of hyperglycemia during enteral and parenteral nutrition therapy. Curr Diab Rep. 2013;13:155-62.

11. Handelsman Y, Mechanick JI, Blonde L, et al. American Association of Clinical Endocrinologists medical guidelines for clinical practice for developing a diabetes mellitus comprehensive care plan. Endocr Prac. 2011;17(Suppl 2):1-53. 